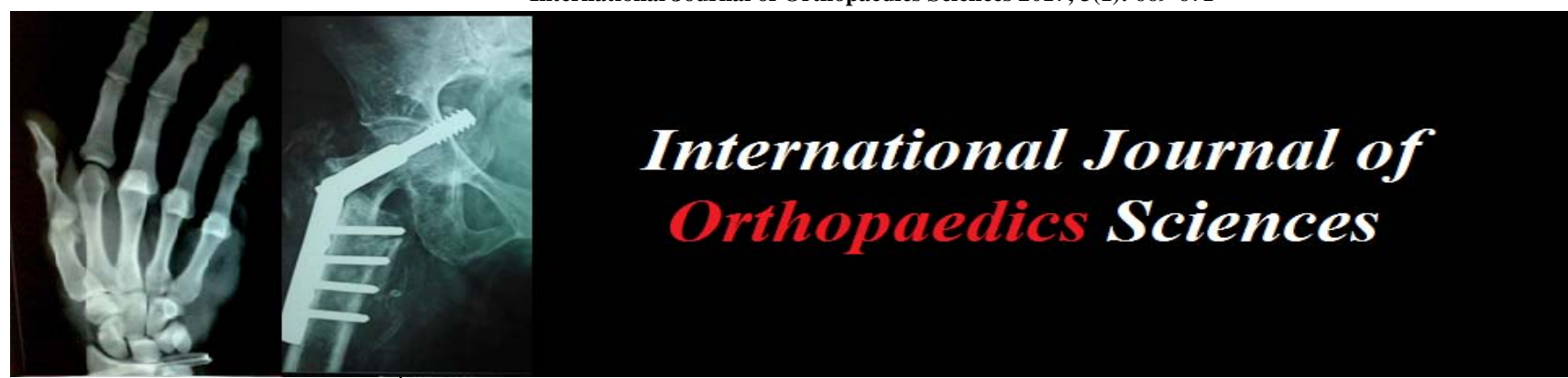

ISSN: $2395-1958$

IJOS 2017; 3(1): 669-671

(C) 2017 IJOS

www.orthopaper.com

Received: 09-11-2016

Accepted: 10-12-2016

Dr. Arunodhaya Siddartha

Assistant Professor, Department

of Orthopaedics, JSS Hospital

Mysore, India

Dr. Supreeth Nekkanti

Senior Resident, Department of

Orthopaedics, JSS Hospital,

Mysore, India

Dr. Vijay C

Associate Professor, Departmen of Orthopaedics, JSS Hospital,

Mysore, India

Dr. Abhilash G

Junior Resident, Department of Orthopaedics, JSS Hospital,

Mysore, India
Correspondence

Dr. Supreeth Nekkanti

Senior Resident, Department of

Orthopaedics, JSS Hospital,

Mysore, India

\section{An unusual presentation of tubercular dactylitis with subluxation of the metacarpo-phalangeal joint in an adult}

\author{
Dr. Arunodhaya Siddartha, Dr. Supreeth Nekkanti, Dr. Vijay C and \\ Dr. Abhilash G
}

DOI: http://dx.doi.org/10.22271/ortho.2017.v3.i1j.99

\section{Abstract}

Introduction: Tuberculosis rarely affects the bones of the hand and feet. This entity is described as tubercular dactylitis (TB dactylitis). It affects children. TB dactylitis in an otherwise healthy adult is extremely rare.

Case report: We report an extremely rare presentation of TB dactylitis is a 35 year old female patient. She presented to us with pain and swelling of her index finger since a year. A synovial biopsy was taken and diagnosis of TB dactylitis was confirmed. The patient was administered one year of Anti tubercular treatment and was disease free at the end of treatment.

Conclusion: TB dactylitis is extremely rare in adults. There are reports in adults with pre-existing conditions such as HIV or other immunosuppressive conditions. It is the recommendation of the authors not to rule out Tubercular dactylitis as a differential when dealing with chronic painful swelling of fingers in adults.

Keywords: Tubercular dactylitis, adult, subluxation, metacarpo-phalangeal joint

\section{Introduction}

Osteoarticular involvement of Tuberculosis (TB) forms 1-3\% of all forms of extra-pulmonary tuberculosis. ${ }^{[1,2]}$ Tuberculosis involving the small tubular bones of hand and feet is described as tubercular dactylitis (TB dactylitis). TB dactylitis affects the bones of the hand more often than those of the feet. It has a predilection for the proximal phalanx of index and middle fingers. ${ }^{[1,3]}$ TB dactylitis or spina ventosa occurs in usually in children less than six years of age. ${ }^{[1,4]}$ TB dactylitis in adults is extremely rare. ${ }^{[1,2,3]}$ We report an unusual presentation of TB dactylitis affecting the second metacarpal in an adult female patient.

\section{Case Report}

We report a case of a 35 year old female who presented to us with pain and swelling over her right index finger since one year. She was apparently normal one year back following which she developed a vague pain and minimal swelling over her right index finger. She took analgesics for the pain and came to us when the swelling gradually progressed to the present size.

On clinical inspection, the second finger appeared diffusely swollen, with the skin shiny over the entire finger. (Figure.1, 2) On palpation, there was tenderness elicited over the second metacarpal and proximal phalanx. The swelling was doughy in nature, non-fluctuant and nonmobile. The movement of the second metacarpo-phalangeal joint was extremely painful.

Radiography of the hand revealed gross articular erosions of the second metacarpo-phalangeal (MCPJ) joint. There was joint line sclerosis and gross reduction in joint space evident at the $2^{\text {nd }}$ MCPJ. The second metacarpal appeared sclerosed in relation to the phalanges. There was juxta-articular erosion seen at the neck of second proximal phalanx and the neck of $2^{\text {nd }}$ metacarpal. The oblique view showed signs of subluxation and instability of the second MCPJ. (Figure.3) 
After the above evaluation, a provisional diagnosis of tubercular dactylitis was considered.

The patient underwent a subtotal synovectomy of the $2^{\text {nd }}$ MCPJ and loose bodies were removed. The MCPJ was transfixed with a K-wire in view of its instability. (Figure.4) A biopsy of the excised synovial tissue at the second MCPJ was taken.

Histopathology: The tissue exhibited villous hyperplasia. The stroma was infiltrated by lymphoplasmocytes. There were occasional ill-defined aggregates of epitheloid cells and multinucleate giant cells. There was evidence of focal necrosis. Articular cartilage, dead bone and loose fibrocartilaginous bodies seen. A diagnosis of tubercular synovitis of right index finger was made. (Figure.5,6) The patient was pain free and had functional movements of the $2^{\text {nd }} \mathrm{MCPJ}$ at the end of one year. She was free of any disease activity.

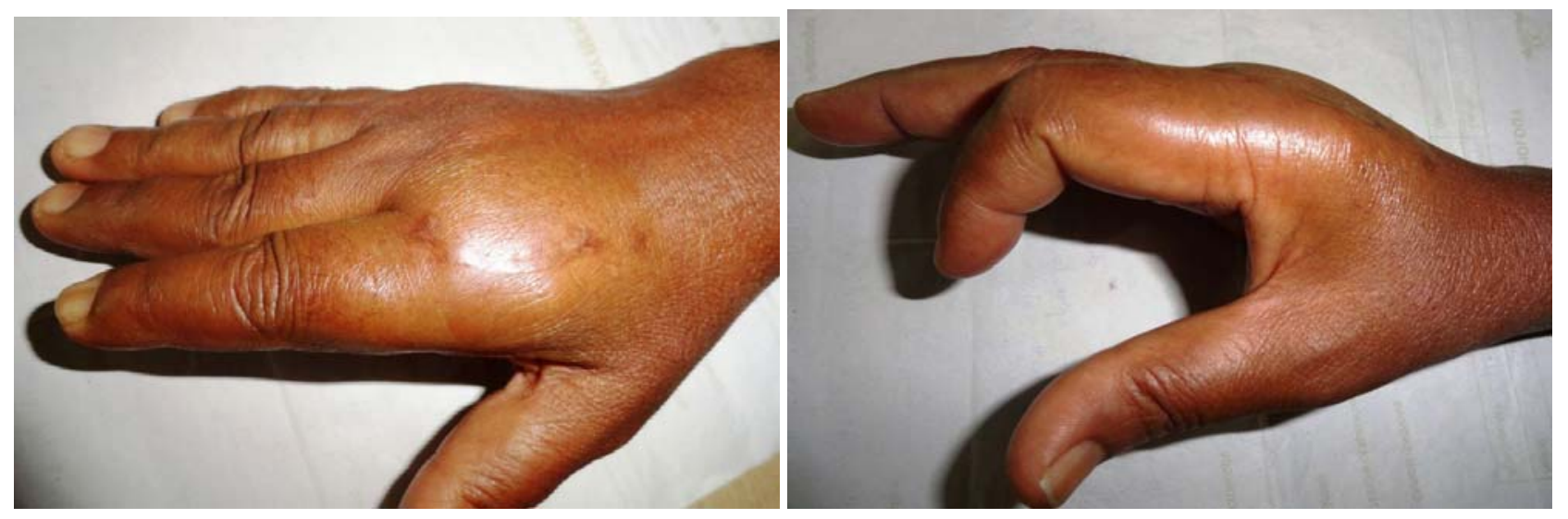

Fig 1, 2: Clinical photo showing TB dactylitis of index finger

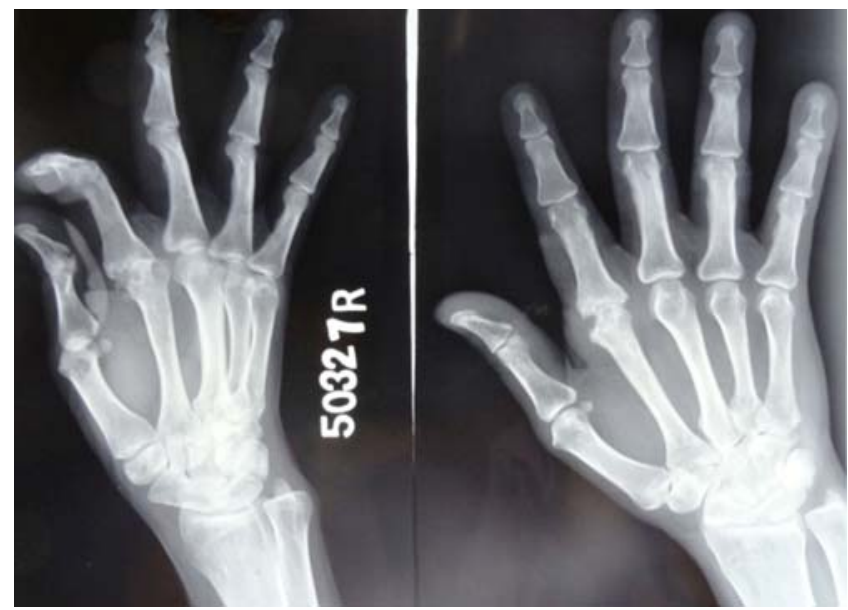

Fig 3: X-ray of hand showing features of TB Dactlitis of $2^{\text {nd }}$ MCPJ

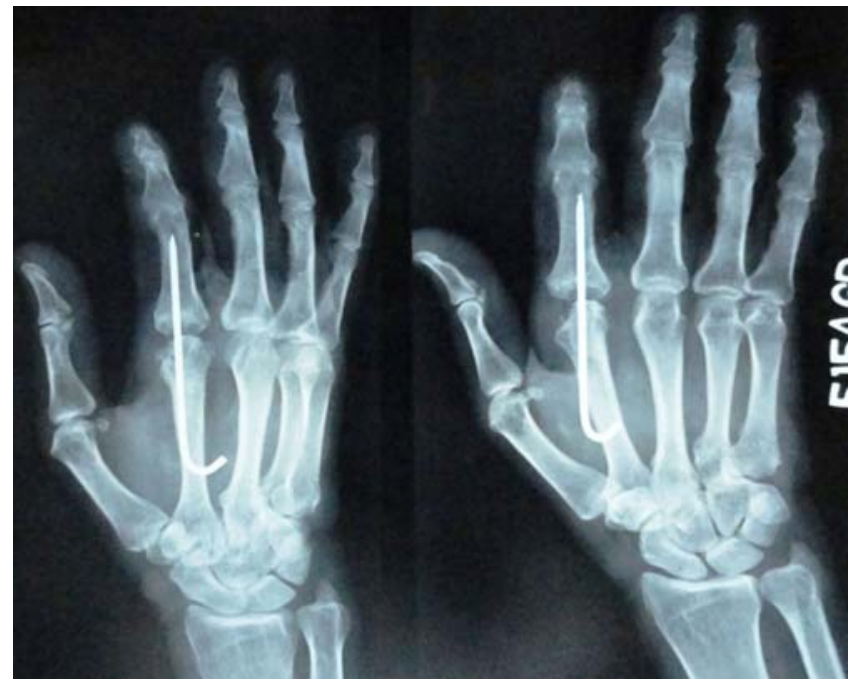

Fig 4: Post-operative x-ray
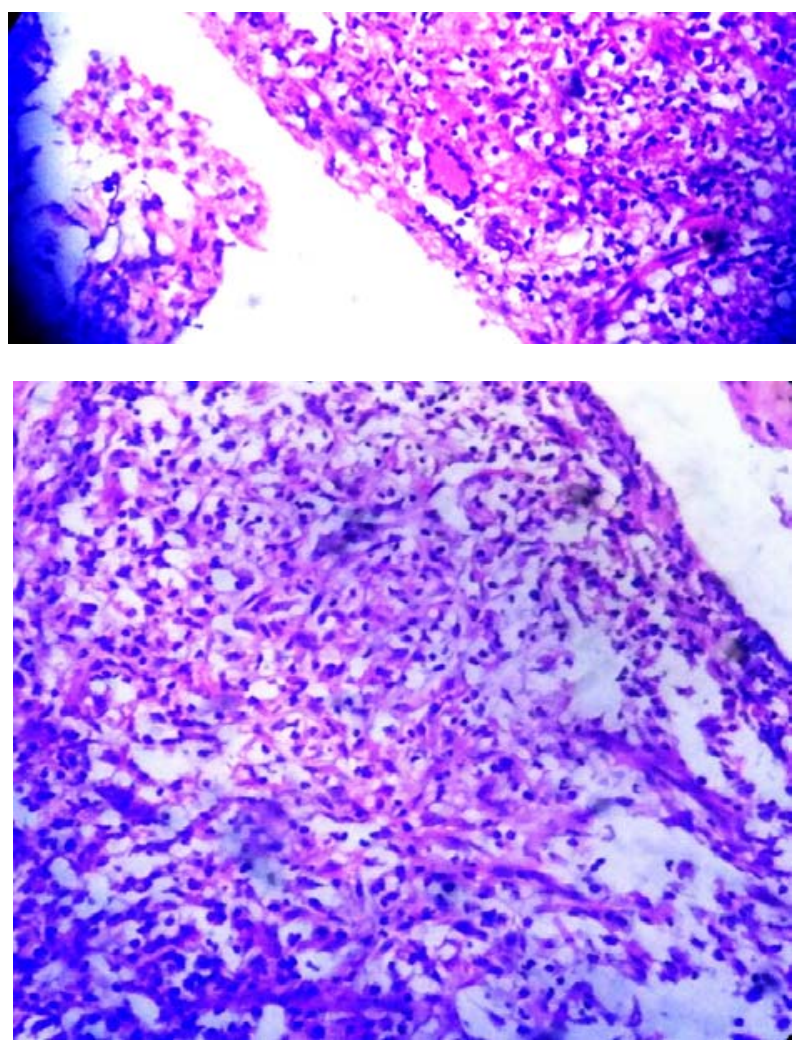

Fig 5, 6 Histopathology photographs

\section{Discussion}

The most common site of osteoarticular Tuberculosis (TB) is the spine. The incidence of TB spine is more than $50 \%$ among all patients with extra-pulmonary TB. ${ }^{[1,5]} \mathrm{TB}$ of small bones like metacarpals and phalanges are extremely rare ${ }^{[1,2]}$ Such cases in which TB affects small bones of hand and feet are labeled as Tubercular dactylitis or spina ventosa based on the clinical presentation. TB dactylitis or spina ventosa occurs in usually in children less than six years of age. ${ }^{[1,4]}$ TB dactylitis in adults is extremely rare. ${ }^{[1-3]}$ The reason for this unusual 
presentation in children is due to the rich blood supply of the small bones by a large nutrient artery which enters into the middle of these small bones. In case of contact with Mycobacterium tuberculae, the inoculums of infection enter these small bones through the nutrient artery and lodges right in the middle of the marrow of the bone. The inoculums progresses to develop into a tubercular granuloma which causes the short tubular bone to gradually enlarge to accommodate the granuloma. This leads to a spindle or fusiform shaped thickening of the short tubular bone which is termed as "spina ventosa". [7] As the tubercular granuloma grows the nutrient artery gets occluded and consequently the inner bony lamella gets destroyed to form a sequestrum. The normal course of TB dactylitis is to heal naturally with shortening and deformity of the affected digit. [7] In our patient, the severe pain in movements and progressive swelling led her to consult our orthopedic team and hence was diagnosed and managed without shortening of the digit. However movements of the $2^{\text {nd }} \mathrm{MCPJ}$ were restricted.

Diagnosis of TB dactylitis is like all other forms of TB, by radiography and culture of mycobacterium tuberculae from the synovial tissue. Although many methods such as Mantoux test, PCR etc exist to diagnose TB, however synovial biopsy remains the best modality to diagnose and confirm TB.

Osteoarticular TB can be diagnosed by radiography also. The commonly seen features are lytic lesion, articular erosions, juxta-articular osteopenia, cysts, joint space reduction, and cysts. [1, 8, 9] Our patient's radiographs showed articular erosions, joint space reduction and extra-articular lytic lesions. There was sclerosis of the second metacarpal which was probably due to sequestrum formation following destruction of internal lamella of the second metacarpal.

Current recommendations for the treatment of osseous tuberculosis include a 2-month initial phase of isoniazid, rifampin, pyrazinamide, and ethambutol followed by a 6-to 12month regimen of isoniazid and rifampin ${ }^{[1,10]}$.

Some schools of thought argue that the paucibacillary nature of the lesion make a 6-month treatment course appropriate. Subasi, et al. ${ }^{[1,11]}$ describe the treatment and outcome of a series of 7 patients with tuberculosis of the metacarpals and phalanges. These patients were treated with a 4-drug regimen for 2 months, followed by a 2-drug regimen for 10 months. Within 6

weeks, patients demonstrated clinical signs of healing and improvement in joint motion. Upon final evaluation (mean follow-up 30 months), all patients had healed lesions without recurrence and none required arthrodesis. In our patient we administered four drug regimen consisting of four drugs isoniazid, rifampin, pyrazinamide, and ethambutol for one year.

In our review of literature, there have been no cases reported in otherwise healthy adults. Few cases have been reported in patients with HIV or as a part of disseminated TB. Possible differential diagnosis of adults presenting with pain and swelling of fingers with no history of trauma, include enchondroma, chronic pyogenic osteomyelitis or syphilitic dactlyitis. In western population diagnosis of sarcoidosis or brucellosis may also be considered. Pyogenic osteomyleitis is more aggressive in its course and is often a very painful condition with discharging sinuses. Enchondromas usually can be diagnosed by radiography and biopsy of the lesion. ${ }^{[7]}$ Our patient was symptomatic for more than a year with progressive swelling and a provisional diagnosis of tubercular dactlyitis was made and confirmed by synovial biopsy. She was disease free after one year of Anti tubercular treatment. She had functional range of movements of her finger with no pain.

\section{Conclusion}

Isolated extra-pulmonary TB dactylitis is extremely rare in adults. TB dactylitis usually presents in young children less than six years of age. We report this case for its unusual age of presentation and rarity. The authors strongly recommend considering TB dactylitis as a possible differential diagnosis of painful finger with swelling. TB dactylitis if left untreated or misdiagnosed leads to severe deformity and shortening of the digit and eventual loss of function of the hand.

\section{Clinical Message}

Spine is the most common site for extrapulmonary TB. TB affecting the small bones of hand and feet is rare and has been reported in children younger than six years of age. TB of the hand or foot has been described in literature either due to dissemination of existing pulmonary tuberculosis or reactivation of a latent focus of TB in the lung. Few cases have been described secondary to pre-existing immunosuppressive disorders like HIV. This case highlights the possibility of a healthy patient with no previous history of tuberculosis or comorbdities to suffer from a primary tuberculous dactylitis. The authors strongly feel that it should be thought of as a differential diagnosis while dealing with patients having chronic painful finger with progressive swelling.

\section{References}

1. Shradha Agarwal, Daniel Caplivski, Edward J. Bottone Disseminated tuberculosis presenting with finger swelling in a patient with tuberculous osteomyelitis: a case report Annals of Clinical Microbiology and Antimicrobials. 2005; 4:18.

2. Evanchick CC, Davis DE, Harrington TM. Tuberculosis of peripheral joints: an often missed diagnosis. J Rheumatol. 1986; 13:187-191.

3. Andronikou S, Smith B. Spina ventosa-tuberculous dactylitis. Archives of Disease in Childhood 2002; 86:206.

4. Karanas YL, Yim KK. Mycobacterium tuberculosis infection of the hand: a case report a and review of the literature. Ann Plast Surg. 1998; 40:65-67.

5. Daniel TM, DebaBanne SM. The serodiagnosis of tuberculosis and other mycobacterial diseases by enzymelinked immunosorbent assay. Am Rev Respir Dis. 1987; 135:1137.

6. Karanas YL, Yim KK. Mycobacterium tuberculosis infection of the hand: a case report and review of the literature. Ann Plast Surg. 1998; 40:65-67.

7. Ranadeb Bandyopadhyay, Arindam Mukherjee, Rajib Kumar Mondal. Case Report: "Spina Ventosa" Tuberculous Dactylitis in a 2 Year Old Boy -A Very Rare Disease The Open Orthopaedics Journal 2012; 6:118-120.

8. Weaver P, Lifeso RM. The radiological diagnosis of tuberculosis of the adult spine. Skel Radiol. 1984; 12:178186.

9. Versfeld GA, Solomon A. A diagnostic approach to tuberculosis of bones and joints. J Bone and Joint Surg. 1982; 64-B(4):446-449.

10. Center for Disease Control and Prevention: Treatment of Tuberculosis. MMWR Morb Mortal Wkly Rep 2003; 52(RR11):1-77.

11. Subasi M, Bukte Y, Kapukaya A, Gurkan F. Tuberculosis of the metacarpals and phalanges of the hand. Ann Plast Surg. 2004; 53(5):469-72. 